\title{
Validation of WHO-IMNCI Algorithm for Jaundice in 0-2 Months Aged Infants at Tertiary Level Hospital
}

\author{
Kanodia $\mathrm{P}^{1}$, Mahaseth $\mathrm{S}^{1}$, Paranjuli $\mathrm{V}^{1}$
}

\section{ABSTRACT}

Introduction: For the effective management of these major childhood illnesses, WHO and UNICEF have developed the "Integrated Management of Neonatal and Childhood IIIness" (IMNCI) Strategy. Aim: The aim of study is to evaluate the utility of the WHO/ UNICEF algorithm for Integrated Management of Neonatal and Childhood Illness (IMNCI) for jaundice up to two months of age. Methods: This is Prospective observational comparative study. Total of 300 subjects were taken from Emergency and Outpatient Department of Pediatrics. The treatment steps were identified as according to the 'Assess and Classify' module of IMNCl algorithm. All relevant investigations were performed, using appropriate methods. Blood sugar was done in all recruited children and serum bilirubin levels were done in all infants presented with jaundice. Based on this detailed clinical evaluation and relevant investigations, final diagnosis were made and therapies were given. These diagnosis and treatments were considered as the 'Gold Standard' for comparison. Results: There were 300 young infants, of whom 162(54\%) were male and 138(46\%) were female infants. Total of 146 infants were admitted, 24 from OPD and 122 from Emergency. 154 infants were sent home after initial management in hospital. Severe jaundice was present in 24 infants according to IMNCl and 12 infants according to Gold Standard in 0-2 months of age. The predictive utility of algorithm for the diagnosis of severe jaundice with a sensitivity of $100 \%$, specificity of $75 \%$, positive predictive value of $50 \%$ and negative predictive value of $100 \%$ in $0-2$ months of age group. Conclusion: Algorithm performed well in identifying severe jaundice with the sensitivity of $100 \%$ and specificity of $95 \%$.

\section{Keywords: Childhood illness, Jaundice, WHO-IMNC}

\section{Authors:}

1. Dr. Piush Kanodia

2. Dr. Sameer Mahaseth

3. Dr. Vishnu Paranjuli

${ }^{1}$ Department of Pediatrics, Nepalgunj Medical College and Teaching Hospital, Nepalgunj, Banke,

\section{Address for Correspondence:}

Dr. Piush Kanodia

Associate Professor

Department of Pediatrics

Nepalgunj Medical College and Teaching Hospital

Nepalgunj, Banke, Nepal

Email: piushkanodia@yahoo.com

\section{INTRODUCTION}

Many Surveys reveal that many sick children are not properly assessed and treated by these health care providers, and that their parents are poorly advised. ${ }^{1}$ Most of the neonatal deaths occurs during the first week of life, making it the most vulnerable period of life. ${ }^{2}$ Early neonatal period (0-7 days) is the most most vulnerable period and needs to be taken into consideration. ${ }^{3}$ For effective management of major childhood illnesses, WHO and UNICEF have developed the "Integrated Management of Childhood Illness" (IMCI) Strategy. ${ }^{4,5}$ Generic $\mathrm{IMCl}$ program did not initially include newborn less than one week old. There are very few published studies where IMNCI algorithm has been tested in children 7 days -2 months old ${ }^{6,7}$ within Nepal subcontinent. IMNCl is an integrated approach to child health that focuses on the well-being of the whole child. IMNCI aims to reduce death, illness and disability, and to promote improved growth and development among children under 5 years of age. IMNCl includes both preventive and curative elements that implemented by families and communities as well as by health facilities. ${ }^{8}$

Hence this present study therefore was done with the objective to evaluate the validity of $\mathrm{IMNCl}$ algorithm in $0-2$ months of age with special emphasis in early neonatal period.

\section{METHODS}

This study is prospective observational comparative study. It is conducted at Emergency and Outpatient Department of Pediatrics, Nepalgunj Medical College, Nepalgunj. Study Duration was One Year from January 2019 to December 2019. In this study the sample size taken were total of 300 subjects Emergency and Outpatient Department of Pediatrics. The sample size was calculated to be sufficient to detect a difference 
of $10 \%$ in diagnostic agreement form the gold standard with $90 \%$ power and an alpha of 0.05 .

Inclusion criteria: Any infant presented with a fresh episode of illness in the Emergency or the Outpatient Department of the Pediatrics in NGMC was included.

Exclusion criteria: Infants attended the well-baby clinic or immunization clinic for routine visits and follow-up care were kept out of the study.

Data collection: For all the children in the study, detailed history and clinical examination listed in $\mathrm{IMNCl}$ algorithm, in the same order. The treatment steps were identified as according to the 'Assess and Classify' module of IMNCl algorithm and was recorded in the proforma. The study subjects so selected were managed according to the protocol of treating unit under the supervision of the senior faculty. All relevant investigations were performed, using appropriate methods. Blood sugar was done in all recruited children and serum bilirubin levels were done in all infants presented with jaundice. Based on this detailed clinical evaluation and relevant investigations, final diagnosis were made and therapies were given. These diagnosis and treatments were considered as the 'Gold Standard' for comparison. The study children were either admitted or sent home after initial evaluation, depending upon the nature and severity of illness. The hospitalized subjects were followed up till the final outcome. Feeding counseling was given to every child with low birth weight or feeding problems. Consent: A written informed consent was taken from all the parents of babies enrolled in the study Data analysis: The data was evaluated in the predesigned proforma, data were entered into MS Excel and analyzed using SPSS 20.01 for windows 7, chi square test and fisher's exact test, sensitivity, specificity, positive predictive value and negative predictive value.

\section{RESULTS}

A total of 300 infants between 0-2 months who fulfilled the study criteria were studied. The baseline profile of study infants is as follows. There were 300 young infants, of whom $162(54 \%)$ were male and $138(46 \%)$ were female infants as shown in Table I.

\begin{tabular}{|ccc|}
\hline Gender & Number & Percent (\%) \\
\hline Male & 162 & $54 \%$ \\
\hline Female & 138 & $46 \%$ \\
\hline Total & 300 & $100 \%$ \\
\hline
\end{tabular}

Table I : Sex distribution of study infants (0-2 months)

\begin{tabular}{|cccc|}
\hline & Admitted & Sent home & Total \\
\hline OPD & 24 & 126 & 150 \\
\hline Emergency & 122 & 28 & 150 \\
\hline Total & 146 & 154 & 300 \\
\hline
\end{tabular}

Table II : Relationship between recruitment and hospitalization (0-2 months)
Out of 300 young infant, 162 infants were 0-7 days old and remaining 138 were in the age group from 7 days upto two months and mean age is 13.27 days which is depicted in Table III.

\begin{tabular}{|c|c|c|}
\hline Duration & Number & Percent \\
\hline $0-7$ days & 162 & $54 \%$ \\
\hline 7 days- 2 months & 138 & $46 \%$ \\
\hline Total & 300 & $100 \%$ \\
\hline
\end{tabular}

\section{Utility of Algorithm to Identify Jaundice}

The agreement between the $I \mathrm{MNCl}$ algorithm and Gold Standard in identification of jaundice is as follows Severe jaundice was present in 24 infants according to IMNCl and 12 infants according to Gold Standard in 0-2 months of age as depicted in Table IV.

\begin{tabular}{|c|cc|}
\hline & IMNCI & Gold Standard \\
\hline No Jaundice & 208 & 228 \\
\hline Jaundice & 68 & 60 \\
\hline Severe Jaundice & 24 & 12 \\
\hline Total & $\mathbf{3 0 0}$ & $\mathbf{3 0 0}$ \\
\hline
\end{tabular}

Table IV : Accuracy of algorithm to identify jaundice (0-2 months)

The predictive utility of algorithm for the diagnosis of severe jaundice with a sensitivity of $100 \%$ and specificity of $75 \%$ in $0-2$ months of age group as shown in Table V.

\begin{tabular}{|cc|}
\hline Sensitivity & $100 \%$ \\
\hline Specificity & $95 \%$ \\
\hline Positive predictive value & $50 \%$ \\
\hline Negative Predictive Value & $100 \%$ \\
\hline Table V : Utility to identify severe jaundice (0-2 months)
\end{tabular}

Table VI and VII shows accuracy of algorithm and its utility respectively for severe jaundice in infants 0-7 days of age. Severe jaundice was present in 18 infants according to IMNCI and 10 infants according to Gold Standard in 0-7 days of age as depicted in Table VI.

\begin{tabular}{|c|cc|}
\hline & IMNCI & Gold Standard \\
\hline No Jaundice & 114 & 116 \\
\hline Jaundice & 30 & 36 \\
\hline Severe Jaundice & 18 & 10 \\
\hline Total & 162 & 162 \\
\hline
\end{tabular}

Table VI : Accuracy of algorithm to identify jaundice (0-7 days)

The predictive utility of algorithm for the diagnosis of severe jaundice with a sensitivity of $100 \%$ and specificity of $94.7 \%$ in 0-7 days of age group was found as shown in Table VII. 


\begin{tabular}{|cc|}
\hline Sensitivity & $100 \%$ \\
\hline Specificity & $94.7 \%$ \\
\hline Positive predictive value & $55.5 \%$ \\
\hline Negative Predictive Value & $100 \%$ \\
\hline
\end{tabular}

Table VII : Utility of identity severe jaundice (0-7 days)

Table VIII and IX shows accuracy of algorithm and its utility respectively for severe jaundice in infants 7-59 days of age Severe jaundice was present in 6 infants according to $\mathrm{IMNCl}$ and 2 infant according to Gold Standard in 7-59 days of age as depicted in Table VIII.

\begin{tabular}{|c|cc|}
\hline & IMNCI & Gold Standard \\
\hline No Jaundice & 94 & 112 \\
\hline Jaundice & 38 & 24 \\
\hline Severe Jaundice & 6 & 2 \\
\hline Total & 138 & 138 \\
\hline
\end{tabular}

Table VIII : Accuracy of algorithm to identify jaundice (7-59 days)

\begin{tabular}{|c|c|}
\hline Sensitivity & $100 \%$ \\
\hline Specificity & $97 \%$ \\
\hline Positive predictive value & $33 \%$ \\
\hline Negative Predictive Value & $100 \%$ \\
\hline
\end{tabular}

Table IX : Utility to identify severe jaundice (7-59 days)

\section{DISCUSSION}

The $I \mathrm{MCl}$ strategy promotes the accurate identification of childhood illness in the outpatient settings, ensures, appropriate combined treatment of all major illnesses, strengthens the counselling of caretakers and the provision of preventive services, and speeds up the referral of severely ill children. ${ }^{9,10}$ This information contributed to the development of the Integrated Management of Childhood Illness (IMCI) algorithms during the mid1990s, which standardized the management of sick young infants at first-level health facilities. ${ }^{11}$

A total of 300 infants between 0-2 months who fulfilled the study criteria were investigated. Of these $162(54 \%)$ were 0-7 days and 138 (46\%) were 7 days -2 months of age. Thus, a considerable number of study infants were between 0-7 days of age to allow us to see whether the $1 \mathrm{MNCl}$ algorithm which has additionally included early neonatal period (0-7 days), can be utilized with equal efficacy in 0-7 days as in 7 days- 2 months age group. About $46 \%$ of study subjects were females and $54 \%$ were males.

\section{Relationship between Recruitment Site and Hospitalization}

Out of 300 young infants, 150 infants were recruited both form OPD and emergency of hospital. Out of 150 infants recruited form OPD $16.0 \%$ were admitted and remaining $84.0 \%$ were sent home after initial evaluation. The corresponding figures form emergency was 81.35 and $18.7 \%$ respectively.
Similar study conducted by Kaur et al ${ }^{12}$ which showed that $97 \%$ of infants coming to emergency were admitted and rest sent home as compared to $54 \%$ admission from OPD. Thus, hospitalization rates among those who presented to the emergency were much higher than those who attended OPD suggesting that a significantly higher proportion of severely ill patients reported to emergency. However, amongst the patients in early neonatal period (0-7 days), $28 \%$ of infants presenting to OPD were hospitalized and $72 \%$ were sent back home. Corresponding figures for infant presenting to emergency was $80.3 \%$ and $19.7 \%$ respectively.

Goswami et $\mathrm{al}^{3}$ showed that $66 \%$ of infants in early neonatal period (0-7 days) were admitted as compared to $52 \%$ in 7 days -2 months age groups. Gupta et al ${ }^{6}$ showed that 595 of infants were admitted and rest $41 \%$ were sent back home in 7 days -2 months age groups. Neonates less than 7 days of age brought to the hospital were sicker and were hospitalized when they presented to OPD and emergency. Out of 300 infants, according to $\mathrm{IMNCl}$ and Gold Standard severe jaundice was present in $24(8 \%)$ and $12(4 \%)$ respectively. Severe Jaundice had high sensitivity of $100 \%$ and specificity of $95 \%$. Study conducted by Kaur et al ${ }^{12}$ showed that according to gold standard jaundice was present in $50.6 \%$ in $0-7$ days and $17.3 \%$ in 7 to 59 days infants. In study conducted by Bhattacharya et al. according to $\mathrm{IMNCl}$ Jaundice and severe jaundice was present in $6 \%$ infants and $1.7 \%$ infants respectively as compared to $34 \%$ and $8 \%$ respectively in the present study. In this study $7.7 \%$ infants had jaundice according to gold standard as compared to $20 \%$ in the present study. Sensitivity and Specificity to identify jaundice were $66.67 \%$ and $99.07 \%$ as compared to $100 \%$ and $95 \%$ in the present study.$^{13}$ According to bicentric study in Bolivia conducted by Mazzi et al jaundice was present as per IMNCl in 0-7 days and 7-59 days as $49 \%$ and $32.8 \%$ infants as compared to $29 \%$ and $31 \%$ in the present study respectively. In this study jaundice as per gold standard, present in 0-7 days and $7-59$ days infants was $55 \%$ and $38.5 \%$ infants as compared to $28 \%$ and $18.8 \%$ in the present study respectively. ${ }^{14}$ This high diagnosis of jaundice according to $\mathrm{IMNCl}$ and gold standard was similar to that in the present study.

In study conducted by Thummakomma $\mathrm{M}$ et al jaundice was present according to gold standard in 0-7 days as $54.2 \%$ and 7-59 days as $45.7 \%$ compared to $28 \%$ and $18.8 \%$ in the present study respectively. According to $\mathrm{IMNCl}$ jaundice was present $19.8 \%$ as compared to $30.6 \%$ in the present study. Sensitivity, specificity, Positive predictive value and negative predictive value in study conducted by Thummakomma $\mathrm{M}$ et al and in the present study was $61.3 \%, 95 \%, 81.4 \%, 94.6 \%$ and $100 \%, 95 \%$, $50 \%, 100 \%$ respectively which is comparable.$^{15}$ 


\section{LIMITATION}

IMNCI Algorithm performed well in identifying severe jaundice and is important tool to diagnose severe jaundice by health care professional. To increase the power of study sample size should be increased and follow up should be done.

\section{CONCLUSION}

The present study was done to validate the IMNCI algorithm in young infants 0-2 months of age. Of the total 300 infants studied $162(54 \%)$ were $0-7$ days of age and 138 were 7 days2 months of age. Seventy-five infants were recruited both from OPD and emergency. As per Gold Standard 146(48.7\%) were hospitalized and 154(51.3\%) were sent back home and managed on outpatient basis after initial evaluation. IMNCI algorithm performed well in identifying severe jaundice with the sensitivity of $100 \%$ and specificity of $95 \%$.

\section{REFERENCES}

1. Integrated Management of Childhood Illness (IMCI) Available at https://www.who.int/ maternal_child_adolescent/ topics/ child/imci/en/

2. Lawn JE, Cousens S, Zupan J; Lancet Neonatal Survival Steering Team. 4 million neonatal deaths: when? Where? Why? Lancet. 2005; 365(9462):891-900. doi: 10.1016/S0140-6736(05)71048-5.

3. Goswami V, Dutta AK, Singh V, Chandra J. Evaluation of simple clinical signs of illness in young infants (0-2 months) and its correlation with $\mathrm{WHO} I \mathrm{MCl}$ algorithm (7 days to 2 months). Indian Pediat. 2006; 43(12):1042-1049.

4. World Health Organization. Integrated management of sick child. Bull WHO 1995; 73:735-740.

5. Gove S. Integrated management of childhood illness by outpatient health workers: technical basis and overview. The WHO Working Group on Guidelines for Integrated Management of the Sick Child. Bull World Health Organ. 1997; 75(1):7-24.

6. Gupta R, Sachdev HPS, Shah D. Evaluation of the WHO/UNICEF algorithm for integrated management of childhood illness between the ages of one week to two months. Indian Pediatric. 2000; 37:383-390.

7. Kalter HD, Schillinger JA, Hossain M, Burnham G, Saha S, de Wit $V$, et al. Identifying sick children requiring referral to hospital in Bangladesh. Bull World Health Organ. 1997; 75(1):65-75.

8. What is Integrated Management of Childhood Illness (IMCI)? Available at https://www.who.int/maternal_child_adolescent/ child/imci/background/en/.

9. Madhu Gupta, Arun Kumar Aggarwal. Feasibility study of $I \mathrm{MNCl}$ guidelines on effective breastfeeding in a rural area of north India. Ind J Comm Med. 2008; 33 (3):201-203. doi: 10.4103/0970-0218.42067.

10. Patwari AK, Raina N. Integrated Management of Childhood Illness (IMCI): a robust strategy. Ind J Pediat. 2002; 69(1):41-48. doi: https://doi.org/10. 1007/ BF02723776.
11. Tulloch J. Integrated approach to child health in developing countries. The Lancet. 1999; 354:SII16-20. doi: https://doi. org/10.1016/S0140-6736(99)90252-0.

12. Kaur S, Singh V, Dutta AK, Chandra J. Validation of $I \mathrm{MNCl}$ algorithm for young infants (0-2 months) in India. Indian Pediatr. 2011; 48(12):955-960. Epub 2011 Mar 15. doi: 10.1007/s13312011-0155-1.

13. Bhattacharyya A, Mukherjee S, Chatterjee C, Dasgupta S. Assessment of validity and reliability of $\mathrm{IMNCl}$ algorithm in comparison to provisional diagnosis of senior pediatricians in a tertiary hospital of Kolkata. J Fam Med Primary care. 2013; 2(2):173-177. doi: 10.4103/2249-4863.117393.

14. Mazzi E, Bartos AE, Carlin J, Weber MW, Darmstadt GL, Bolivia Clinical Signs Study Group. Clinical signs predicting severe illness in young infants ( $<60$ days) in Bolivia. Journal of tropical pediatrics. 2010 Feb 8; 56(5):307-316. doi: https://doi.org/10. 1093/ tropej/fmq005.

15. Thummakomma M, Ramesh P, Reddy M, Balram B. Utility of implementing integrated management of neonatal and childhood illness (IMNCI) algorithm in a tertiary care hospital for the young infants of age 0-2 months. J Evid Med Healthcare. 2016;3(89):4868-4872. doi: 10.18410/jebmh/2016/1026. 